\title{
BMJ Open Effects of advanced life support versus basic life support on the mortality rates of patients with trauma in prehospital settings: a study protocol for a systematic review and meta-analysis
}

\author{
Yutaka Kondo, ${ }^{1}$ Tatsuma Fukuda, ${ }^{1}$ Ryo Uchimido, ${ }^{2}$ Toru Hifumi, ${ }^{3}$ Kei Hayashida ${ }^{4}$
}

To cite: Kondo Y, Fukuda T, Uchimido R, et al. Effects of advanced life support versus basic life support on the mortality rates of patients with trauma in prehospital settings: a study protocol for a systematic review and meta-analysis. BMJ Open 2017;7:e016912. doi:10.1136/ bmjopen-2017-016912

- Prepublication history and additional material for this paper are available online. To view please visit the journal (http:// dx.doi.org/10.1136/bmjopen2017-016912).

Received 23 March 2017 Revised 16 August 2017 Accepted 25 August 2017

\section{CrossMark}

${ }^{1}$ Department of Emergency and Critical Care Medicine, Graduate School of Medicine, University of the Ryukyus, Okinawa, Japan ${ }^{2}$ Department of Emergency Medicine, Mie Prefectural Shima Hospital, Mie, Japan

${ }^{3}$ Emergency Medical Center, Kagawa University Hospital,

Kagawa, Japan

${ }^{4}$ Department of Emergency and Critical Care Medicine, School of Medicine, Keio University, Tokyo, Japan

Correspondence to

Dr. Yutaka Kondo;

kondokondou2000@yahoo.co.jp

\section{ABSTRACT}

Introduction Advanced life support (ALS) is thought to be associated with improved survival in prehospital trauma care when compared with basic life support (BLS). However, evidence on the benefits of prehospital ALS for patients with trauma is controversial. Therefore, we aim to clarify if ALS improves mortality in patients with trauma when compared with BLS by conducting a systematic review and meta-analysis of the recent literature. Methods and analysis We will perform searches in PubMed, Embase and the Cochrane Central Register of Controlled Trials for published observational studies, controlled before-and-after studies, randomised controlled trials and other controlled trials conducted in humans and published until March 2017. We will screen search results, assess study selection, extract data and assess the risk of bias in duplicate; disagreements will be resolved through discussions. Data from clinically homogeneous studies will be pooled using a random-effects meta-analysis, heterogeneity of effects will be assessed using the $\chi^{2}$ test of homogeneity, and any observed heterogeneity will be quantified using the $\mathrm{I}^{2}$ statistic. Last, the Grading of Recommendations Assessment, Development and Evaluation approach will be used to rate the quality of the evidence.

Ethics and dissemination Our study does not require ethical approval as it is based on findings of previously published articles. Results will be disseminated through publication in a peer-reviewed journal, presentations at relevant conferences and publications for patient information.

Trial registration number PROSPERO (International Prospective Register of Systematic Reviews) registration number CRD42017054389.

\section{INTRODUCTION}

Advanced life support (ALS) is widely accepted as the gold standard of prehospital care in patients with cardiopulmonary arrest (CPA) caused by intrinsic diseases. ${ }^{1-3}$ It has also been suggested that ALS in patients with trauma contributes to improved survival. However, it is not known if prehospital ALS
Strengths and limitations of this study

- We will conduct a systematic review and metaanalysis of the effects of advanced life support (ALS) and basic life support (BLS) on survival in patients with trauma in prehospital settings using appropriate methodologies and quality assessment tools.

- We will also perform subgroup analysis by trauma type to evaluate the efficacies of ALS versus BLS; this will provide further clinical evidence for clinicians and patients.

- The results of this systematic review and metaanalysis will be highly dependent on the quality of the included primary research studies; many observational studies might be included.

is more beneficial for patients with trauma than basic life support (BLS). In previous studies, prehospital ALS increased the time that was spent on the scene and thus delayed definitive in-hospital care. ${ }^{4}$ In contrast, prehospital BLS consists of non-invasive interventions that are easy to perform, require little added on-scene time and can often be performed en route by minimally trained emergency medical staff. The treatment of trauma is time-sensitive; thus, rapid transportation to the hospital is required as in-hospital surgery is typically needed to improve the prognosis of patients with trauma. ALS might put patients with trauma at risk as it increases the time spent on-scene; however, providing ALS can also be used to resuscitate patients with trauma on-scene. In 2017, von Vopelius-Feldt and colleagues ${ }^{6}$ reported in their systematic review that prehospital critical care had a limited effect; none of the included studies showed obvious benefits for non-trauma CPA patients. This implies that it is important to treat patients, and particularly patients with trauma, at the hospital rather 
than in prehospital settings. Resources are very limited in prehospital settings.

In 2016, Evans et $a l^{7}$ reported that prehospital ALS procedures in patients with traumatic cardiac arrest were not associated with increased odds of survival using two large registry data sets (Resuscitation Outcomes Consortium Epistry-Trauma and the Prospective Observational Prehospital and Hospital Registry for Trauma registries). Endotracheal intubation in prehospital settings has not been shown to reduce mortality and morbidity in patients with severe trauma; moreover, there are concerns that performing this difficult task under trying conditions might cause harm. ${ }^{8-11}$ Endotracheal intubation by unskilled practitioners could result in adverse events and result in ineffective chest compressions with significant interruptions. ${ }^{8}$ The value of prehospital intravenous fluid resuscitation has also been questioned. ${ }^{12-14}$ Intravenous fluids given before surgical control of bleeding lead to either accentuation of ongoing haemorrhage or hydraulic disruption of an effective thrombus, followed by a fatal secondary haemorrhage. In addition, intravenous infusions of crystalloid may promote haemorrhage by diluting coagulation factors and by lowering blood viscosity. ${ }^{12}$ In summary, suitable interventions for trauma care in prehospital settings are still controversial.

Therefore, we aim to clarify if ALS improves mortality in patients with trauma when compared with BLS by conducting a systematic review and meta-analysis of the recent literature.

\section{METHODS AND ANALYSIS}

This systematic review and meta-analysis protocol has been registered in PROSPERO, an International Prospective Register of Systematic Reviews at the National Institute for Health Research and Centre for Reviews and Dissemination at the University of York (http://www.crd.york.ac. uk/PROSPERO/; registration no CRD42017054389)..$^{15}$ The protocol follows the Preferred Reporting Items for Systematic Reviews and Meta-Analyses Protocols (PRISMA-P) statements (online supplementary file 1), ${ }^{16} 17$ and the systematic review and meta-analysis will be reported in accordance with PRISMA guidelines. ${ }^{16} 1819$

\section{Types of studies}

Observational studies, controlled before-and-after studies (CBAs; studies with contemporaneous data collection before and after an intervention), randomised controlled trials (RCTs) and other controlled trials (CTs; studies that are not truly randomised but have treatment allocations) will be included. Conference abstracts, studies in animals and those that only include patients with trauma transported by helicopter will be excluded.

\section{Study population}

Our study population of interest is adult ( $>18$ years old) patients with trauma who were transported by ground transportation and required resuscitation in prehospital settings. We will not restrict our analysis by country and will include all severities and types of trauma. Data on patients without signs of life and studies with participants aged $\leq 18$ years will be excluded.

\section{Intervention types}

The interventions of interest are ALS and BLS. ALS will be defined as one or more of the following intervention components: (1) tracheal intubation, (2) needle tracheostomy, and administration of (3) intravenous fluids, (4) epinephrine or (5) other intravenous drugs (eg, amiodarone, lidocaine or magnesium). BLS will be defined as BLS procedures only.

\section{Outcomes}

Our primary outcomes of interest are survival to hospital admission and survival to hospital discharge. Secondary outcomes include neurological outcomes and adverse events/complications (including failure rates of ALS and time spent on scene).

\section{Database searches}

Database searches will be conducted in Medline (via PubMed), Embase and the Cochrane Central Register of Controlled Trials to retrieve relevant articles for the literature review. We will search for full-text observational studies, CBAs, RCTs and other CTs in humans that were published until March 2017 in all languages. We will attempt to translate non-English-language articles by using professional translators, if possible. We will consult a librarian for conducting the database searches. Search terms will include 'Trauma', 'Advanced Cardiac Life Support', 'Basic Life Support', 'Life Support Care', 'Emergency Medical Services', 'First Aid' and 'Resuscitation'. We will use a combination of these key terms and establish a full search strategy (online supplementary file 2).

\section{Data extraction and management}

The following data will be extracted: author(s), title, journal name, year of publication, website (URL) and abstract. The authors (YK, TF and RU) will perform a first-line comprehensive literature search independently and blindly followed by filtering for duplicates. After removal of duplicates by two of the three authors, titles and abstracts will be screened independently and blindly for potential relevance using a pretested electronic screening form (Covidence web platform: http://www. COVIDENCE.org). In cases of disagreements among the reviewers, the full text of the paper will be retrieved; disagreements will be reconsidered and discussed until a consensus is reached. If disagreements cannot be reconciled, a third reviewer will be consulted. Inter-reviewer agreement will be assessed through the Cohen's $\kappa$ coefficient. ${ }^{20}{ }^{21}$ For this statistic, values $<0.4$ indicate poor, 0.4-0.59 fair, 0.60-0.74 good and $>0.75$ excellent agreement. $^{22}$

The full text of the articles included in the final selection will be independently reviewed by two authors, who 


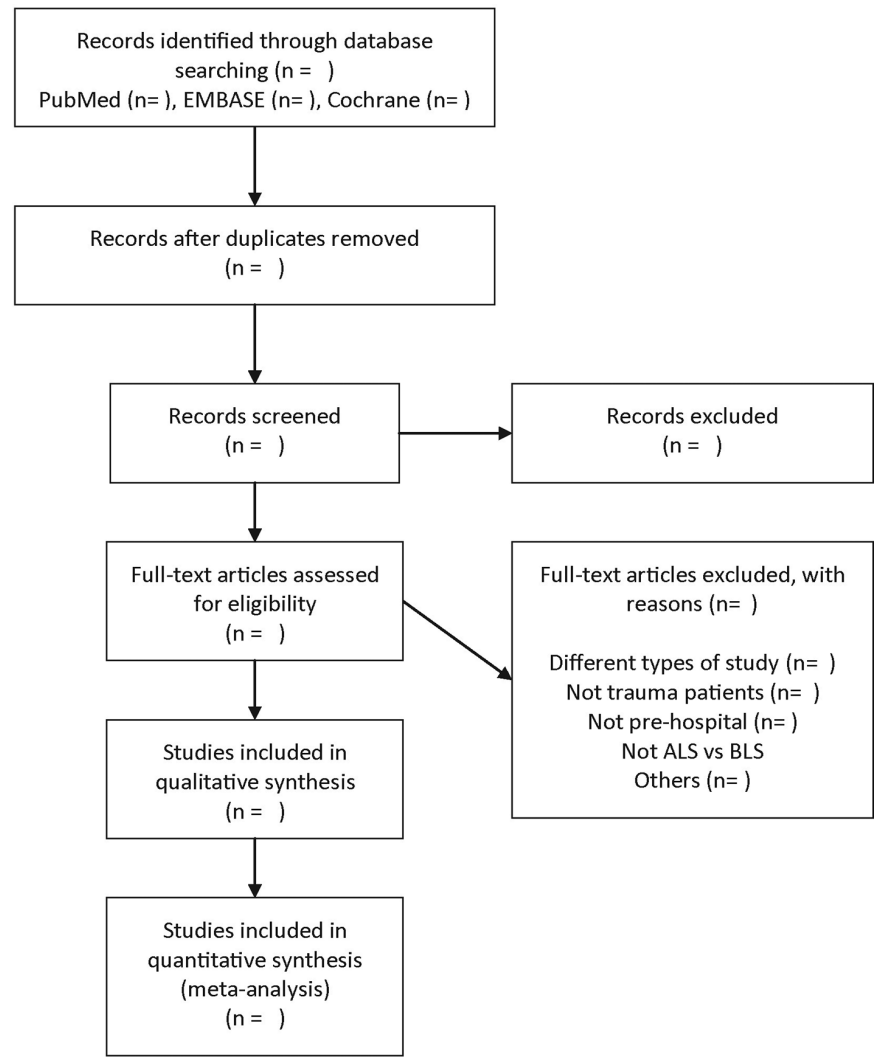

Figure 1 Flow chart of the study selection process. ALS, advanced life support; BLS, basic life support.

will be randomly chosen from five authors (YK, TF, RU, $\mathrm{TH}$ and $\mathrm{KH}$ ). The flow diagram of our study, which has been adapted from the PRISMA statement $(2009),{ }^{19}$ is shown in figure 1.

\section{Assessment of risk of bias}

To assess the quality of the included studies, we will adapt the Cochrane risk of bias tool. ${ }^{23}$ Each study will be assessed for (1) random sequence generation (selection bias), (2) allocation concealment (selection bias), (3) blinding of participants and personnel (performance bias), (4) blinding of related outcomes assessment (detection bias), (5) incomplete outcome data (attrition bias), (6) selective reporting (reporting bias) and (7) other bias. Studies will be categorised as having a low, unclear or high risk of bias in each domain. The risk of bias for each element will be considered 'high' when bias is present and likely to affect outcomes, and 'low' when bias is not present or present but unlikely to affect outcomes. ${ }^{24}$

Two independent reviewers, chosen from the five authors (YK, TF, RU, TH and KH), will perform the risk of bias assessment. Disagreements will be resolved through discussion; a third reviewer will provide his/her opinion, if necessary.

\section{Summarising data}

We plan to perform a meta-analysis when data are available in one or more trials according to the 'Cochrane Handbook for Systematic Reviews of Interventions' and the PRISMA guidelines by using the Review Manager software (RevMan V.5.3, Copenhagen, Denmark: The Nordic Cochrane Centre, The Cochrane Collaboration 2014). Results will be summarised using the generic inverse variance method to facilitate pooling of estimates of the treatment effects.

OR and 95\% CIs will be used for dichotomous outcomes, and mean differences or standardised mean differences and $95 \%$ CIs for continuous outcomes, when appropriate. If quantitative synthesis is not appropriate for a particular outcome, we will provide a qualitative summary for this outcome. ${ }^{25}$

\section{Assessment of heterogeneity}

Heterogeneity between trials for each outcome will be evaluated using the $\mathrm{I}^{2}$ statistic for quantifying inconsistency. ${ }^{26}$ We will consider heterogeneity as being significant if the reason for heterogeneity cannot be explained, and if $\mathrm{I}^{2}$ is $50 \%$ or greater. If significant heterogeneity is found, the median of the estimates rather than a weighted pooled estimate will be reported. Clinical heterogeneity will be explored by assessing differences in baseline data, types of trauma, ALS definitions and other outcome parameters. The presence of strong clinical heterogeneity will be considered in the decision to conduct a quantitative synthesis of data or to perform sensitivity analyses with a special focus. ${ }^{27}$

\section{Assessment of reporting bias}

We will investigate the potential for publication bias using a funnel plot. To test for funnel plot asymmetry, we will apply the Egger and arcsine tests for continuous and dichotomous outcomes, respectively, using the STATA SE V.13 statistical software. ${ }^{28} 29$

\section{Data synthesis}

Estimates will be pooled using a random-effects model. We will attempt to contact the primary authors of the publications for additional data, if possible. In detail, we will email the authors and wait for a response for 1 week. If we do not receive a response by then, we will send another email. We will not use data that include identifiable information. The meta-analysis will be performed based on all published data and data made available to us. ${ }^{24}$ We do not plan to perform multiple imputation for missing data.

\section{Subgroup analyses}

Subgroup analyses will be used to investigate the differences in pooled effect estimates related to different patient subgroups included in the studies. We will evaluate if a differential intervention effect among the various subgroups exists by using an interaction analysis, as this is preferred over separate subgroup group-specific analyses. Subgroup analyses will be performed for types of trauma (blunt vs penetrating), rural versus urban, and by prehospital procedures. Moreover, we will categorise the studies by time of publication (published in the last 10 years vs 
published 10 years ago or longer) and analyse potential differences between the two groups.

\section{Sensitivity analysis}

To ensure the robustness of evidence, we will perform sensitivity analysis to assess the effect of studies with a high risk of bias. We will compare the results to decide whether lower quality studies should be excluded based on sample size, strength of evidence or effect on pooled effective size. Moreover, we might consider a leave-one-out sensitivity meta-analysis if a study shows big population and looked different types of study. ${ }^{30}$

\section{Rating the quality of evidence using the GRADE approach}

Two authors (randomly chosen from YK, TF, RU, TH and $\mathrm{KH})$ will independently use the Grading of Recommendations Assessment, Development and Evaluation (GRADE) tool to rate the quality of the evidence on the effect of ALS and BLS on important outcomes in patients with trauma. ${ }^{31-34}$ Although the quality of evidence represents a continuum, it will be assessed for each outcome and categorised as high, moderate, low or very low using the GRADEpro Guideline Development Tool.

\section{DISCUSSION}

The benefits of ALS in patients with trauma in prehospital settings have not been clearly established yet. ${ }^{35-37}$ Thus, we aim to clarify if ALS increases survival when compared with BLS in prehospital trauma care by conducting a systematic review and meta-analysis of the recent literature. Importantly, we will exclude patients with trauma transported by helicopter from our analyses. Excluding these patients will likely result in more accurate data when compared with previously published meta-analyses, as helicopter transportation differs from a typical prehospital setting (eg, it is difficult to perform chest compressions in a flying helicopter).

To the best of our knowledge, only two meta-analyses reported data on ALS versus BLS in patients with trauma thus far. In 2000, Liberman $e t a l^{38}$ showed that ALS was associated with an increased mortality rate (2.5 times) when compared with BLS. Moreover, the time spent on scene was higher for ALS than for BLS providers $(18.5 \mathrm{~min} \text { vs } 13.5 \mathrm{~min} \text {, respectively; } \mathrm{p}=0.005)^{38}$; this can affect mortality. In a study by Bakalos et $a l^{39}$ in 2011, ALS care was not associated with increased survival. The authors retrieved data from nine trials including 16857 patients who met their inclusion criteria. ${ }^{11} 40-47$ ALS care in patients with trauma was shown to reduce the probability of survival to hospital discharge by almost $10 \%$ when compared with BLS care (pooled OR 0.892, 95\% CI 0.775 to 1.026$).{ }^{39}$ However, this study included only few CTs of sufficient quality and strength that examined the survival of patients with trauma that were published at the time.

Since then, more publications on ALS have become available, and transportation systems and care for patients have been improved. Our study will include all types of trauma as ALS and BLS are performed independent of the type of trauma. We will also perform subgroup analysis by trauma type to evaluate the efficacies of ALS and BLS; this can be a more informative study because it has been argued a lot for several decades. As the effectiveness of prehospital care depends on the transportation method that is used ${ }^{48}$ studies with helicopter transportation will be excluded from our meta-analysis due to the potential risk of bias. In contrast to BLS, most patients with trauma receiving ALS are transported by helicopter. Moreover, the ALS group might include patients with more severe trauma who will also have a worse prognosis (as previous meta-analyses have reported). Our meta-analysis will be the first to exclude patients transported by helicopter transportation, which might result in novel findings. This systematic review and meta-analysis will provide current evidence for researchers in this field and be helpful for clinical staff in treating patients with trauma.

Contributors YK and TF conceived the idea for this systematic review. YK, TF, RU, TH and KH developed the methodology. The manuscript was drafted by YK. TF, RU, $\mathrm{TH}$ and $\mathrm{KH}$ supported in revising the manuscript. All authors critically reviewed and approved the final manuscript.

Funding This study was supportedby grant from JSPS KAKENHI Grant Number $16 K 20394$ (Y.K.).

Competing interests None declared.

Provenance and peer review Not commissioned; externally peer reviewed.

Open Access This is an Open Access article distributed in accordance with the Creative Commons Attribution Non Commercial (CC BY-NC 4.0) license, which permits others to distribute, remix, adapt, build upon this work non-commercially, and license their derivative works on different terms, provided the original work is properly cited and the use is non-commercial. See: http://creativecommons.org/ licenses/by-nc/4.0/

(c) Article author(s) (or their employer(s) unless otherwise stated in the text of the article) 2017. All rights reserved. No commercial use is permitted unless otherwise expressly granted.

\section{REFERENCES}

1. Ocal O, Ozucelik DN, Avci A, et al. A comparison of the outcome of CPR according to AHA 2005 ACLS and AHA 2010 ACLS guidelines in cardiac arrest: multicenter study. Int J Clin Exp Med 2015;8:21549-56.

2. Schneider T, Mauer D, Diehl P, et al. Quality of on-site performance in prehospital advanced cardiac life support (ACLS). Resuscitation 1994;27:207-13.

3. Neumar RW, Otto CW, Link MS, et al. Part 8: adult advanced cardiovascular life support: 2010 American heart association guidelines for cardiopulmonary resuscitation and emergency cardiovascular care. Circulation 2010;122(18 Suppl 3):S729-S767.

4. Rappold JF, Hollenbach KA, Santora TA, et al. The evil of good is better: Making the case for basic life support transport for penetrating trauma victims in an urban environment. J Trauma Acute Care Surg 2015;79:343-8.

5. Seamon MJ, Doane SM, Gaughan JP, et al. Prehospital interventions for penetrating trauma victims: a prospective comparison between advanced life support and basic life support. Injury 2013;44:634-8.

6. von Vopelius-Feldt J, Brandling J, Benger J. Systematic review of the effectiveness of prehospital critical care following out-of-hospital cardiac arrest. Resuscitation 2017;114:40-6.

7. Evans CC, Petersen A, Meier EN, et al. Prehospital traumatic cardiac arrest: management and outcomes from the resuscitation outcomes consortium epistry-trauma and PROPHET registries. J Trauma Acute Care Surg 2016;81:285-93.

8. Hasegawa K, Hiraide A, Chang Y, et al. Association of prehospital advanced airway management with neurologic outcome and survival in patients with out-of-hospital cardiac arrest. JAMA 2013;309:257-66. 
9. Spaite DW, Criss EA. Out-of-hospital rapid sequence intubation: are we helping or hurting our patients? Ann Emerg Med 2003;42:729-30.

10. Wang HE, Peitzman AB, Cassidy LD, et al. Out-of-hospital endotracheal intubation and outcome after traumatic brain injury. Ann Emerg Med 2004;44:439-50.

11. Stiell IG, Nesbitt LP, Pickett W, et al. The OPALS Major Trauma Study: impact of advanced life-support on survival and morbidity. CMAJ 2008;178:1141-52.

12. Bickell WH, Wall MJ, Pepe PE, et al. Immediate versus delayed fluid resuscitation for hypotensive patients with penetrating torso injuries. N Engl J Med 1994;331:1105-9.

13. Dick WF. Controversies in resuscitation: to infuse or not to infuse (1). Resuscitation 1996;31:3-6.

14. Pepe PE. Controversies in resuscitation: to infuse or not to infuse (2). Resuscitation 1996;31:7-10.

15. Moher D, Booth A, Stewart L. How to reduce unnecessary duplication: use PROSPERO. BJOG 2014;121:784-6.

16. Moher D, Shamseer L, Clarke M, et al. Preferred reporting items for systematic review and meta-analysis protocols (PRISMA-P) 2015 statement. Syst Rev 2015;4:1.

17. Shamseer L, Moher D, Clarke M, et al. Preferred reporting items for systematic review and meta-analysis protocols (PRISMA-P) 2015: elaboration and explanation. BMJ 2015;349:97647.

18. Liberati A, Altman DG, Tetzlaff J, et al. The PRISMA statement for reporting systematic reviews and meta-analyses of studies that evaluate health care interventions: explanation and elaboration. $J$ Clin Epidemiol 2009;62:e1-e34.

19. Moher D, Liberati A, Tetzlaff J, et al. Preferred reporting items for systematic reviews and meta-analyses: the PRISMA statement. PLoS Med 2009;6:e1000097.

20. McGinn T, Wyer PC, Newman TB, et al. Tips for learners of evidencebased medicine: 3 . Measures of observer variability (kappa statistic). CMAJ 2004;171:1369-73.

21. Landis JR, Koch GG. The measurement of observer agreement for categorical data. Biometrics 1977;33:159-74.

22. Urban D, Dehaeck R, Lorenzetti D, et al. Safety and efficacy of tranexamic acid in bleeding paediatric trauma patients: a systematic review protocol. BMJ Open 2016;6:e012947.

23. Higgins JPT AD, Sterne JAC on behalf of the Cochrane Statistical Methods Group and the Cochrane Bias Methods Group. Chapter 8: Assessing risk of bias in included studies, 2011.

24. Duan EH, Oczkowski SJ, Belley-Cote E, et al. $\beta$-Blockers in sepsis: protocol for a systematic review and meta-analysis of randomised control trials. BMJ Open 2016;6:e012466.

25. Kondo Y, Fuke R, Hifumi T, et al. Early rehabilitation for the prevention of postintensive care syndrome in critically ill patients: a study protocol for a systematic review and meta-analysis. BMJ Open 2017;7:e013828.

26. Huedo-Medina TB, Sánchez-Meca J, Marín-Martínez F, et al. Assessing heterogeneity in meta-analysis: $\mathrm{Q}$ statistic or 12 index? Psychol Methods 2006;11:193-206.

27. Klotz R, Klaiber U, Grummich K, et al. Percutaneous versus surgical strategy for tracheostomy: protocol for a systematic review and meta-analysis of perioperative and postoperative complications. Syst Rev 2015;4:105.

28. Sterne JA, Sutton AJ, loannidis JP, et al. Recommendations for examining and interpreting funnel plot asymmetry in meta-analyses of randomised controlled trials. BMJ 2011;343:d4002.

29. Davey Smith G, Egger M. Meta-analyses of randomised controlled trials. Lancet 1997;350:1182.
30. Wang ZY, Zhao K, Zheng J, et al. Systematic review and metaanalysis of the association between complement factor $\mathrm{H} \mathrm{I62V}$ polymorphism and risk of polypoidal choroidal vasculopathy in Asian populations. PLoS One 2014;9:e88324.

31. Atkins D, Eccles M, Flottorp S, et al. Systems for grading the quality of evidence and the strength of recommendations I: critical appraisal of existing approaches The GRADE Working Group. BMC Health Serv Res 2004;4:38.

32. Atkins D, Best D, Briss PA, et al. Grading quality of evidence and strength of recommendations. BMJ 2004;328:1490.

33. Mustafa RA, Santesso N, Brozek J, et al. The GRADE approach is reproducible in assessing the quality of evidence of quantitative evidence syntheses. J Clin Epidemiol 2013;66:736-42.

34. Guyatt GH, Oxman AD, Schünemann HJ, et al. GRADE guidelines: a new series of articles in the Journal of Clinical Epidemiology. J Clin Epidemiol 2011;64:380-2.

35. Carrico CJ, Holcomb JB, Chaudry IH. Scientific priorities and strategic planning for resuscitation research and life saving therapy following traumatic injury: report of the PULSE Trauma Work Group. Post Resuscitative and Initial Utility of Life Saving Efforts. Shock 2002;17:165-8.

36. Lewis RJ. Prehospital care of the multiply injured patient: the challenge of figuring out what works. JAMA 2004;291:1382-4

37. Spaite DW, Criss EA, Valenzuela TD, et al. Prehospital advanced life support for major trauma: critical need for clinical trials. Ann Emerg Med 1998;32:480-9.

38. Liberman M, Mulder D, Sampalis J. Advanced or basic life support for trauma: meta-analysis and critical review of the literature. $J$ Trauma 2000;49:584-99.

39. Bakalos G, Mamali M, Komninos C, et al. Advanced life support versus basic life support in the pre-hospital setting: a meta-analysis. Resuscitation 2011:82:1130-7.

40. Osterwalder JJ. Mortality of blunt polytrauma: a comparison between emergency physicians and emergency medical techniciansprospective cohort study at a level I hospital in eastern Switzerland. $J$ Trauma 2003;55:355-61.

41. Lee A, Garner A, Fearnside M, et al. Level of prehospital care and risk of mortality in patients with and without severe blunt head injury. Injury 2003;34:815-9.

42. Liberman M, Mulder D, Lavoie A, et al. Multicenter Canadian study of prehospital trauma care. Ann Surg 2003;237:153-60.

43. Eckstein M, Chan L, Schneir A, et al. Effect of prehospital advanced life support on outcomes of major trauma patients. J Trauma 2000;48:643-8.

44. Schmidt U, Frame SB, Nerlich ML, et al. On-scene helicopter transport of patients with multiple injuries-comparison of a German and an American system. J Trauma 1992;33-548-55.

45. Garner A, Rashford S, Lee A, et al. Addition of physicians to paramedic helicopter services decreases blunt trauma mortality. Aust N Z J Surg 1999;69:697-701.

46. Hamman BL, Cué JI, Miller FB, et al. Helicopter transport of trauma victims: does a physician make a difference? J Trauma 1991;31:490-4.

47. Potter D, Goldstein G, Fung SC, et al. A controlled trial of prehospital advanced life support in trauma. Ann Emerg Med 1988;17:582-8.

48. Ryynänen OP, lirola T, Reitala J, et al. Is advanced life support better than basic life support in prehospital care? A systematic review. Scand J Trauma Resusc Emerg Med 2010;18:62. 